\title{
Impact of Action Video Gaming Behavior on Attention, Anxiety, and Sleep Among University Students
}

\author{
Fatimah Alsaad', Lujain Binkhamis', Amal Alsalman', Njood Alabdulqader (1D', Mashael Alamer', Turki Abualait², \\ Mohamed S Khalil (iD) ${ }^{3}$, Kholoud S Al Ghamdi (D) ${ }^{4}$ \\ 'College of Medicine, Imam Abdulrahman Bin Faisal University, Dammam, Saudi Arabia; ${ }^{2}$ College of Applied Medical Sciences, Imam Abdulrahman Bin \\ Faisal University, Dammam, Saudi Arabia; ${ }^{3}$ Department of Psychiatry, College of Medicine, Imam Abdulrahman Bin Faisal University, Dammam, Saudi \\ Arabia; ${ }^{4}$ Department of Physiology, College of Medicine, Imam Abdulrahman Bin Faisal University, Dammam, Saudi Arabia \\ Correspondence: Njood Alabdulqader, Tel +966 54355080I, Email njoodella@outlook.sa
}

Background: Recently, there has been an increase in the prevalence of action video gaming among adolescents and young adults. This has made video gaming a topic of interest for behavioral and higher brain cognitive function researchers. The present study investigated the impact and consequences of action video gaming on human behavior — specifically, attention, anxiety levels, and sleep patterns.

Objective: The study aimed to investigate the potential associations between action video gaming and attention, anxiety, and sleep. Methods: Recruited participants $(\mathrm{N}=97)$ were asked to independently complete an online questionnaire consisting of 4 sections: demographic data, gaming behavior, 8-item Epworth Sleepiness Scale, and 7-item Generalized Anxiety Disorders Scale. Participants were further divided into 2 groups (expert and non-expert video gamers) based on the number of hours they spent on action video games. After completing the questionnaires, the patients attended an on-site session, where they completed a validated psychological online battery test that assessed their sustained attention.

Results: The mean age of the participants was 21 years. There was a significant difference in attention between expert and non-expert video gamers; when exposed to stimuli, expert gamers displayed significantly shorter reaction times than the non-expert gamers $(\mathrm{p}<$ 0.05). Both groups showed a non-significant decrease in attention span throughout time. The data demonstrated no statistically significant difference in anxiety levels or daytime sleepiness between expert and non-expert video gamers, and minimal to mild anxiety levels were reported in most expert and non-expert gamers.

Conclusion: Expert video gamers were significantly more attentive compared to non-expert gamers, and most participants showed low levels of generalized anxiety. Accordingly, expanding our knowledge on the effects of action video games on attention span is important for creatively using games in the field of education, especially for those who suffer from attention deficit hyperactivity disorders.

Keywords: games, attention, anxiety, sleep, reaction time

\section{Introduction}

Remarkable recent evolutions in technology have made video gaming one of the most popular entertainment practices. Studies have shown a significant increase in the prevalence of gaming among children and adolescents in recent years. ${ }^{1}$ This has led to many emerging studies on the effects of video gaming, especially for educators, social workers, and researchers. This increasing attention toward video gaming is also due to the variable possible impacts on individual well-being associated with gaming behavior. There are many studies that aimed to determine the prevalence and the correlation of gaming behavior on multiple psychological aspects, particularly on attention. ${ }^{1,2}$ These studies resulted with a prevalence of video gaming addiction ranging from $0.7 \%$ to $15.6 \% .1$ Some studies showed that playing action video games could be associated with improvement of some cognitive functions. ${ }^{3,4}$ Other studies detected poor academic 
performance in individuals with addictive gaming behaviors as well as declines in other cognitive functions compared to individuals with less extensive gaming behavior. ${ }^{2,4}$

In addition to attention, anxiety is a major mental health problem of concern as being commonly associated with video/internet gaming. Many studies have investigated this correlation using various methods. One study showed that people with Internet gaming disorder (IGD) demonstrated abnormal emotional regulation, expressed as anxiety and other psychological symptoms. ${ }^{5}$ Some studies detected an association between anxiety and online gaming and IGD. ${ }^{6}$ Furthermore, this association may not only exist in individuals with IGD; there is a possible correlation between anxiety and video gaming even in average gamers, as anxiety itself is a major concerning symptom that could interprets a person's well-being. ${ }^{7,8}$

In addition, poor sleep quality is one of the most common issue being possible associated with video gaming. Poor sleep quality is known to be associated with various other mental health problems and can contribute to both attention changes and anxiety related to video or internet gaming. ${ }^{9,10}$ Video gaming can also indirectly induce negative physical or psychological effects due to sleep deprivation. ${ }^{10,11}$ Further research is necessary to investigate the association between gaming behavior and sleep. This study aimed to explore the possible associations between action gaming behavior and attention, anxiety, and sleep. It also aimed to explore the relationships between the number of hours spent on video gaming (expert versus non-expert) and attentiveness, anxiety, and sleep disturbances.

\section{Methods}

\section{Ethics Statement}

The study was approved by the Institutional Review Board at Imam Abdulrahman Bin Faisal University (IRB-UGS2020-01-358). Informed consent was obtained from each participant, and confidentiality of the data was assured and complies with the Declaration of Helsinki.

\section{Study Design}

A cross-sectional study was conducted from January 2021 to March 2021 at Imam Abdulrahman Bin Faisal University, Khobar, Saudi Arabia.

\section{Participants}

A total of 97 right-handed healthy participants (34 females and 63 males; age range: $18-26$ years; mean age: $21.3 \pm 1.78$ years) were recruited. All participants completed an electronic questionnaire that was designed and divided into 3 sections. The first section collected demographic data, gaming behavior (gamer/non-gamer and the number of hours spent gaming per week), and contact information (with the full explanatory note containing the ethical requirements and consent form). The next section measured sleep deprivation using the 8-item Epworth Sleepiness Scale. ${ }^{16}$ The final section aimed to evaluate the presence of anxiety symptoms using the 7-item Generalized Anxiety Disorders Scale (GAD-7). ${ }^{14}$ Participants who met the following inclusion criteria were recruited further for an on-site psychological battery test: Healthy adults with no history of neurological, psychiatric, or sleep disorders who reported playing action video games for at least 1 hour per week for the prior 6 months Based on the survey responses, all participants who did not fulfill the criteria were excluded from the study. The rest were classified into "expert" and "non-expert" categories according to the number of hours they had spent playing action video games over the previous 6 months. Expert gamers $(n=55)$ were defined as those who played more than 7 hours per week. Non-expert gamers $(n=42)$ were defined as those who played a maximum of 6 hours per week. ${ }^{12}$

\section{Materials and Methods}

\section{Stimulus and Reaction Time Procedure}

The following task, validated in $2013,{ }^{13}$ was used to assess sustained alertness and attention by measuring repeated reactions to recurrent stimuli. 


\section{Stimulus}

The task comprised letters in the alphabet, shown at a fixed point in the center of the screen, in white on a black background. When the visual stimulus (the letter X) appeared in the center of the circle, participants had to respond by pressing the space bar as quickly as possible. They were not to respond when any other stimulus- $\mathrm{K}, \mathrm{O}, \mathrm{A}, \mathrm{R}$, etc.appeared. ${ }^{13}$

\section{Procedure}

Each participant was seated in front of a laptop in a quiet room and given clear instructions. Prior to beginning the experiment, all participants were familiarized with the task and practiced a minimum of 5 trials to assure that they understood the task.

Both groups of gamers were asked to have adequate sleep based on their norms, not to smoke, and not to ingest caffeine one night before the task. After informed consent was obtained, verbal and written on-screen instructions were given. Then, each trial began with the 1500-ms presentation of a blank circle (no stimulus appeared), indicating that the subjects were to relax. When a stimulus was ready to appear, a cross appeared within the circle for $2000 \mathrm{~ms}$, indicating that a stimulus was going to be presented, followed by presentation of the actual stimulus. Stimuli appeared in a random manner with an inter-stimulus interval (ISI) varying between 2 and 8 seconds, as shown in Figure 1. A total of 37 stimuli were presented. The mean reaction time and number of correct responses were recorded. After completing the task with 37 trials, the participants received a report of their performance, including their reaction time, error, and trial accuracy, in the form of a single Excel sheet. ${ }^{13}$

\section{Anxiety}

To examine the impact of video gaming on anxiety, each healthy participant completed a self-reported online questionnaire. This questionnaire, based on a validated English form of the GAD-7, ${ }^{14}$ was intended to assess and measure general anxiety symptoms rather than to diagnose generalized anxiety disorder GAD. ${ }^{3}$ Participants rated the presence of their anxiety symptoms over the prior 2 weeks according to a 4-point Likert scale - "not at all" (0), "several days" (1), "more than half the days" (2), or "nearly every day" (3). Items were summed to reveal a symptom severity score ranging from 0 to 21 . Participants' anxiety was considered minimal if their GAD score was $0-4$, mild if 5-9, moderate if 10-14, and severe if $15-21 .{ }^{15}$

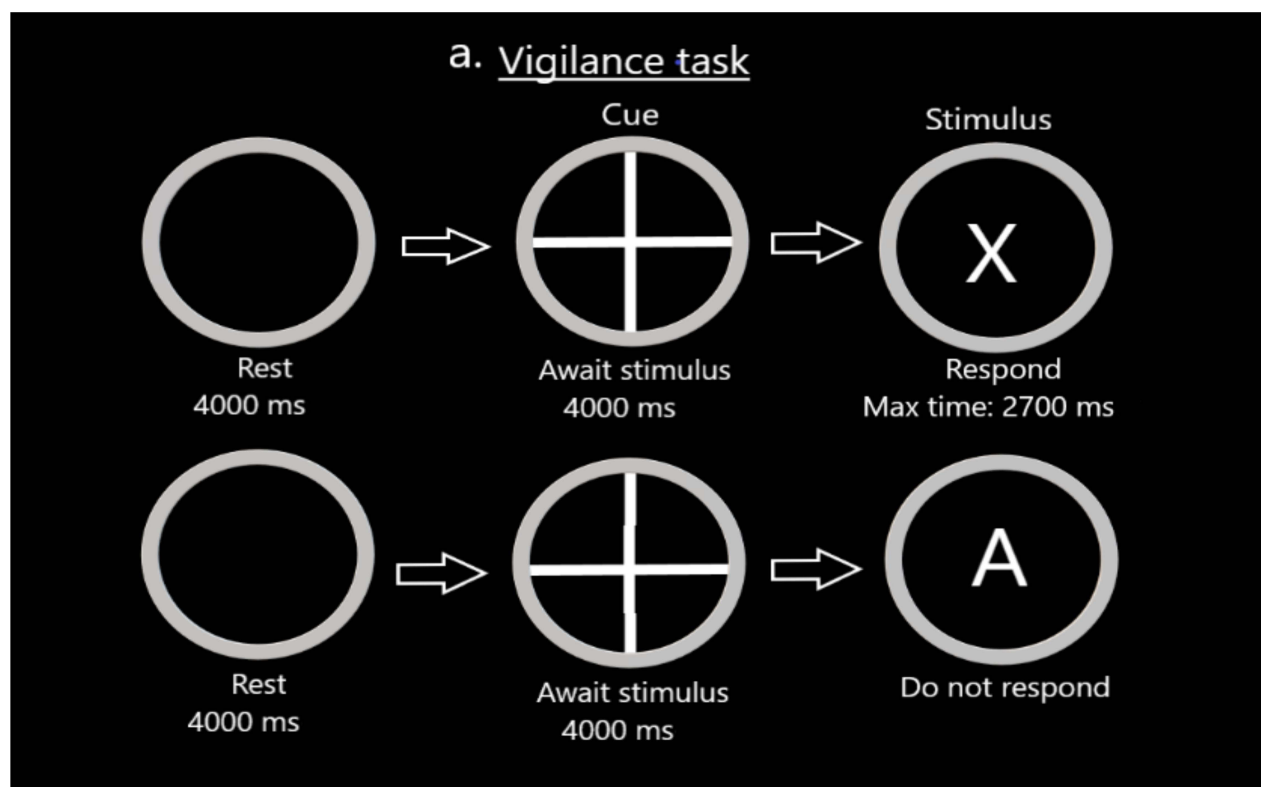

Figure I A sample of the screen as seen by participants. 


\section{Sleep}

A validated English version of the Epworth Sleepiness Scale ${ }^{16}$ was used to explore the effects of action video gaming on daytime sleepiness. This scale consists of the following 7 scenarios: "sitting and reading," "watching TV," "sitting inactive in a public place," "as a passenger in a car for an hour without a break," "lying down to rest in the afternoon when circumstances permit," "sitting and talking to someone," "sitting quietly after lunch without alcohol," and "in a car, while stopped for a few minutes in traffic." For each scenario, sleepiness level was ranked from 0 ("no chance of dozing off") to 3 ("high chance of dozing off"). Participants were identified as experiencing normal daytime sleepiness if their score was $0-10$ and as having excessive daytime sleepiness if their score was $11-24 .^{17}$

\section{Data Analysis}

Data were transferred to a single Excel sheet, and all variables were analyzed using the Statistical Package for the Social Sciences (SPSS) version 26.0. All incorrect responses and non-responses from the attention psychological battery test were deleted. Categorical data were presented as frequencies and percentages. Means with standard deviations (SDs) were calculated for numerical data. Correlations between action video gaming and anxiety and sleep were analyzed using the chi-square test. To illustrate the impact of action video games on sustained attention, we compared the means of the 2 groups using an independent $t$-test. Participants' 37 trials were divided into 5 trial bins, with $7-8$ trials per bin. The mean reaction time for every trial was calculated for all participants. To compare the differences in reaction time span for each trial bin, a one-way analysis of variance (ANOVA) was conducted. A p-value of $\leq 0.05$ was considered statistically significant.

\section{Results}

\section{Demographics}

The total number of study participants was 97 (55 [56.7\%] expert gamers and 42 [43\%] non-expert gamers). Males $(\mathrm{n}=63)$ accounted for $64.9 \%$ of the participants. The mean participant age was $21.01 \pm 1.776$ years.

\section{Gaming and Attention}

Table 1 shows the difference in mean reaction time between expert and non-expert gamers. Expert gamers exhibited significantly shorter reaction times compared to non-expert gamers $(\mathrm{p}=0.013)$, confirming the hypothesis that expert gamers are more attentive than non-experts.

Further analysis was carried out to assess each subject's attention span across all trials. Thirty-seven trials were divided into 5 bins with an average of 7 trials in each. Although no significant differences $(p=0.929)$ were found across all bins according to an ANOVA test, there was an overall trend of reaction times increasing across all bins, as illustrated in Table 2 and Figure 2.

\section{Gaming and GAD}

The prevalence of generalized anxiety symptoms as measured by the validated English version of the GAD-7 and the association with action video games among participants are depicted in Table 3, Figures 3 and 4 . The vast majority of expert (87.2\%) and non-expert (81\%) gamers reported minimal to mild symptoms of anxiety, while $12.7 \%$ of expert gamers and $19.1 \%$ of non-expert gamers reported moderate to severe symptoms of anxiety. There was no statistically significant difference regarding the effects of video gaming on anxiety levels between the 2 groups $(\mathrm{p}=0.388)$.

Table I Reaction Time Comparison Between Expert and Non-Expert Gamers

\begin{tabular}{|l|l|l|l|l|}
\hline Gamers & $\mathbf{n}$ & Mean Reaction Time (ms) & SD & p-value \\
\hline Expert gamers & 55 & 358.24 & 51.90 & 0.013 \\
Non-expert gamers & 42 & 391.20 & 75.48 & \\
\hline
\end{tabular}

Abbreviations: $\mathrm{n}$, number; ms, millisecond; SD, standard deviation. 
Table 2 Attention Span in All Types of Gamers

\begin{tabular}{|c|c|c|c|c|c|c|c|}
\hline \multirow[t]{3}{*}{ Reaction Time } & \multirow{2}{*}{\multicolumn{2}{|c|}{ Total }} & \multicolumn{4}{|c|}{ Group } & \multirow[t]{3}{*}{ p-value } \\
\hline & & & \multicolumn{2}{|c|}{ Expert Gamers } & \multicolumn{2}{|c|}{ Non-Expert Gamers } & \\
\hline & Mean & SD & Mean & SD & Mean & SD & \\
\hline Ist 8 trials & 375.8 & 95.9 & 376.0 & 96.7 & 375.6 & 96.0 & 0.983 \\
\hline 2nd 7 trials & 376.6 & 72.0 & 384.0 & 74.2 & 367.0 & 68.7 & 0.253 \\
\hline 3 rd 8 trials & 362.9 & 54.6 & 354.6 & 46.8 & 373.8 & 62.4 & 0.086 \\
\hline 4th 7 trials & 374.5 & 82.8 & 362.0 & 74.0 & 390.9 & 91.3 & 0.088 \\
\hline 5th 7 trials & 375.9 & 64.2 & 375.0 & 67.6 & 377.1 & 60.3 & 0.873 \\
\hline
\end{tabular}

\section{Gaming and Sleep}

The prevalence of daytime sleepiness among the participants is summarized in Table 4. While most expert gamers (85.5\%) and non-expert gamers (78.5\%) experienced normal daytime sleepiness, $14.5 \%$ of experts and $21.5 \%$ of nonexperts experienced excessive daytime sleepiness. There was no significant association between action video games and severity of daytime sleepiness between expert and non-expert video gamers $(\mathrm{p}=0.377)$.

\section{Discussion}

The present study investigated the possible interrelation between action video games on attention, anxiety, and sleep. Attention is defined as the ability to focus on a specific goal or information while successfully avoiding derailment due to distractions. In that regard, video games channel the facilitation of selective information processing in individuals. Efficient attention is essential in everyday life and is also considered an integral part of action video games. ${ }^{18}$ The current study examined the effects of action video gaming among expert and non-expert gamers on 2 domains of attention. First, players' processing speed of reaction time was measured. Secondly, the reaction time trials were divided into 5 bins to extract the effect of action video games on sustained attention. To ensure elimination of the test's novelty effect, the test trials were complemented with the pre-practice test. Reaction time was found to be superior in the group of expert gamers $(358.25 \mathrm{~ms})$ compared to the non-expert gamers $(391.2 \mathrm{~ms})(\mathrm{p}=0.013)$. This finding is in concordance with a review of studies that tested reaction times between action video game players and non-players and found that the former group had $10 \%$ faster responses compared to the latter group. The study tested the speed of gamers' responses using

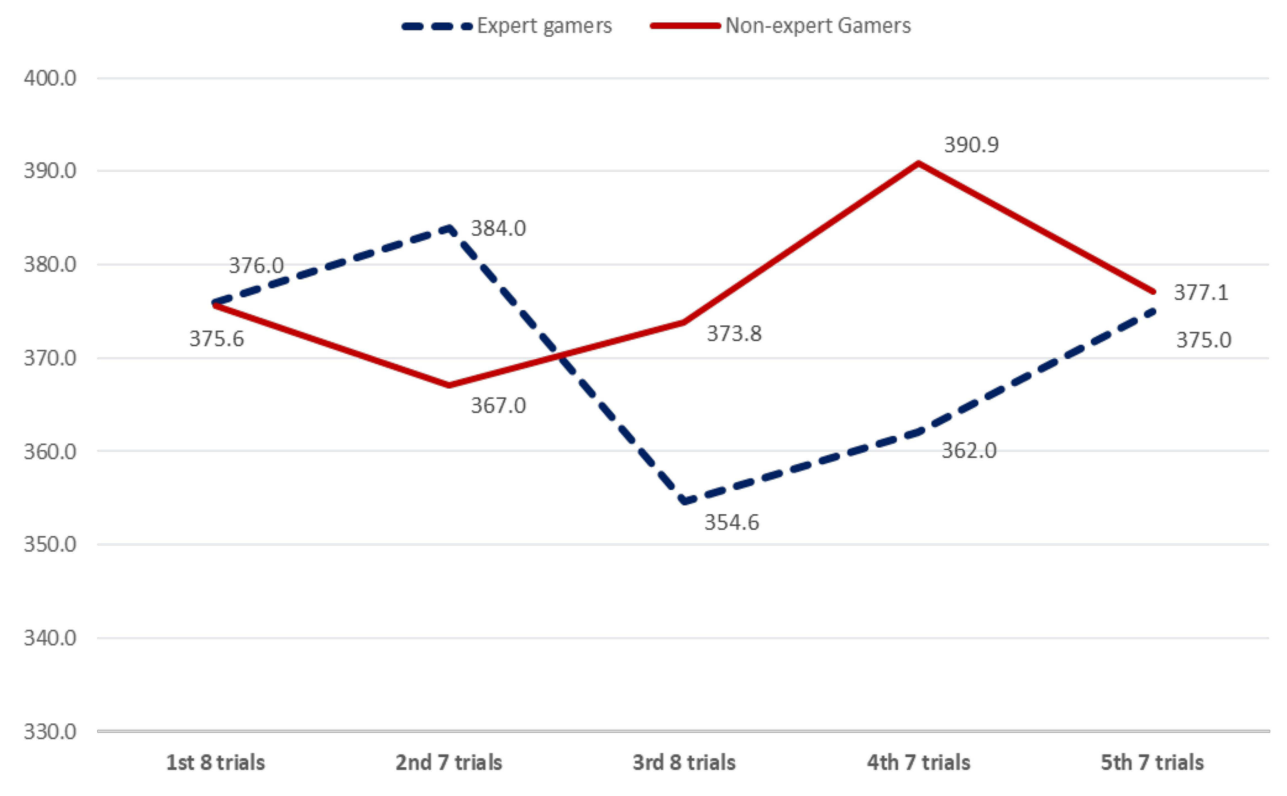

Figure 2 Line chart demonstrating attention span decline in overall gamers. 
Table 3 GAD Levels for Expert and Non-Expert Gamers

\begin{tabular}{|l|l|l|l|l|l|l|}
\hline \multirow{2}{*}{ Gamers } & \multicolumn{2}{|l|}{ n (\%) } & \multirow{2}{*}{ Total (\%) } \\
\cline { 2 - 5 } & Minimal & Mild & Moderate & Severe & \\
\hline Expert gamers & $\mathbf{3 2}(\mathbf{5 8 . 2 \% )}$ & $16(\mathbf{2 9 . 1 \% )}$ & $\mathbf{5}(\mathbf{9 . 1 \% )}$ & $\mathbf{2}(\mathbf{3 . 6 \% )}$ & $\mathbf{5 5}(\mathbf{5 6 . 7 \% )}$ & \\
Non-expert gamers & $17(40.5 \%)$ & $17(40.5 \%)$ & $6(14.3 \%)$ & $2(4.8 \%)$ & $42(43.3 \%)$ & 0.388 \\
\hline
\end{tabular}

built-in data analysis software and found them to be very fast - to the level that the software documented most of their reactions being anticipatory, reaching response times of $200 \mathrm{~ms}$ or less. ${ }^{19}$ Cross-sectional and interventional study designs have investigated net reaction times and reported decreases over time. ${ }^{19-21}$ This net decrease in reaction time corresponding to action video games has also had its effect beyond the games themselves, extending to daily activities. ${ }^{22}$ One study reported that action video games helped dyslexic children increase their reading pace. ${ }^{23}$ Another study found that these games were associated with faster operational skills in surgeons performing laparoscopic surgeries. ${ }^{24}$

In the present study, reaction time was measured using 37 trials. We divided the trials into 5 bins, with an average of 7 trials each, to extract another observation related to attention-attention span. We found no significant difference between the study groups regarding attention span, but we observed a trend of decline in both groups ( $\mathrm{p}=0.93$ ). The effect of action video games on sustaining attention has been extensively studied compared to other aspects of attention. A 2017 study found that expert gamers were better able to maintain attention at the beginning of the task; however, similarly to our study, their performance steadily declined near the end of the task. ${ }^{4}$ The observed similarity of reaction time and the trend of decline in performance throughout the test in both expert and non-expert gamers groups might be

\section{GAD 7-items scale (Expert Gamers)}

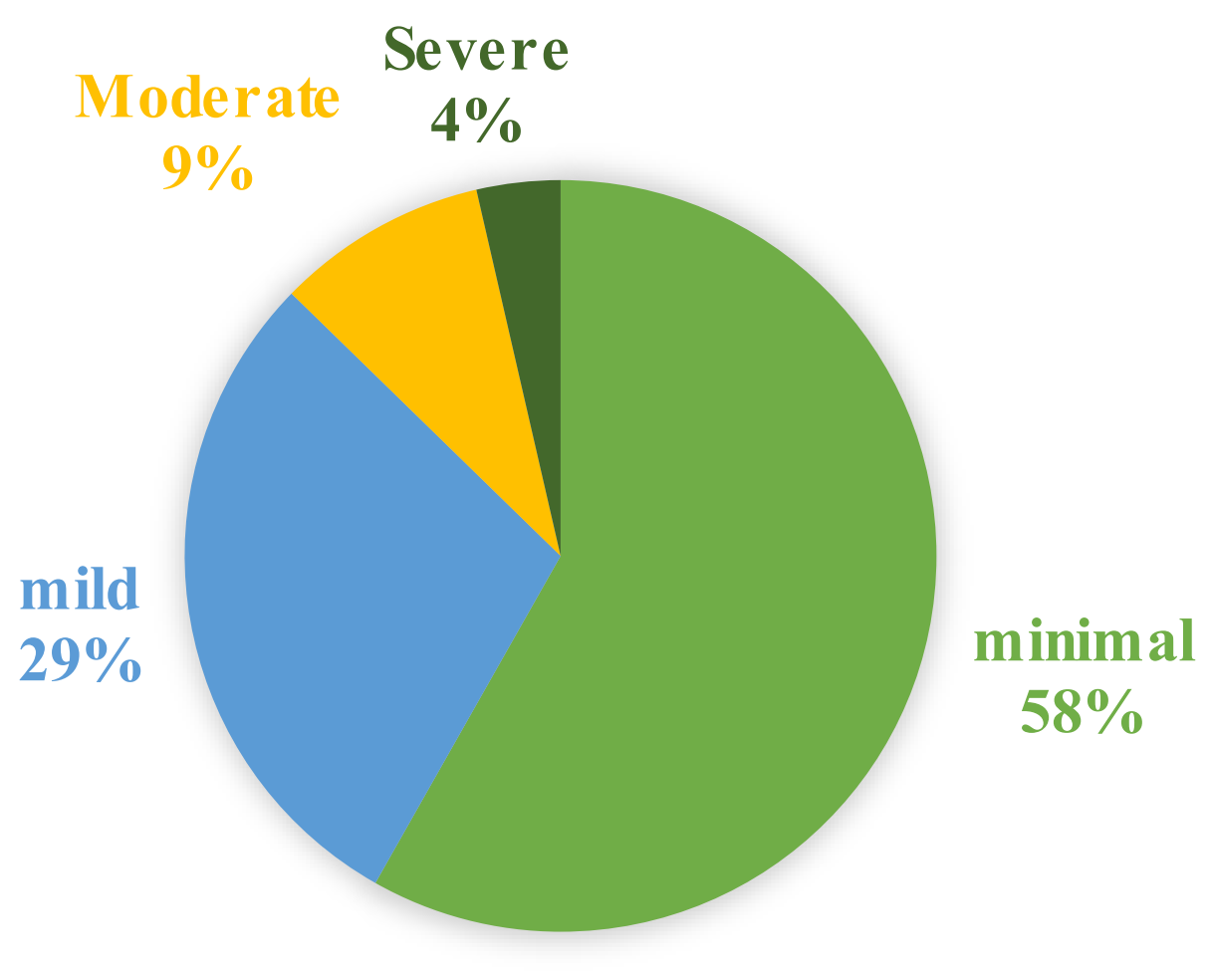

Figure 3 GAD score for expert gamers. 


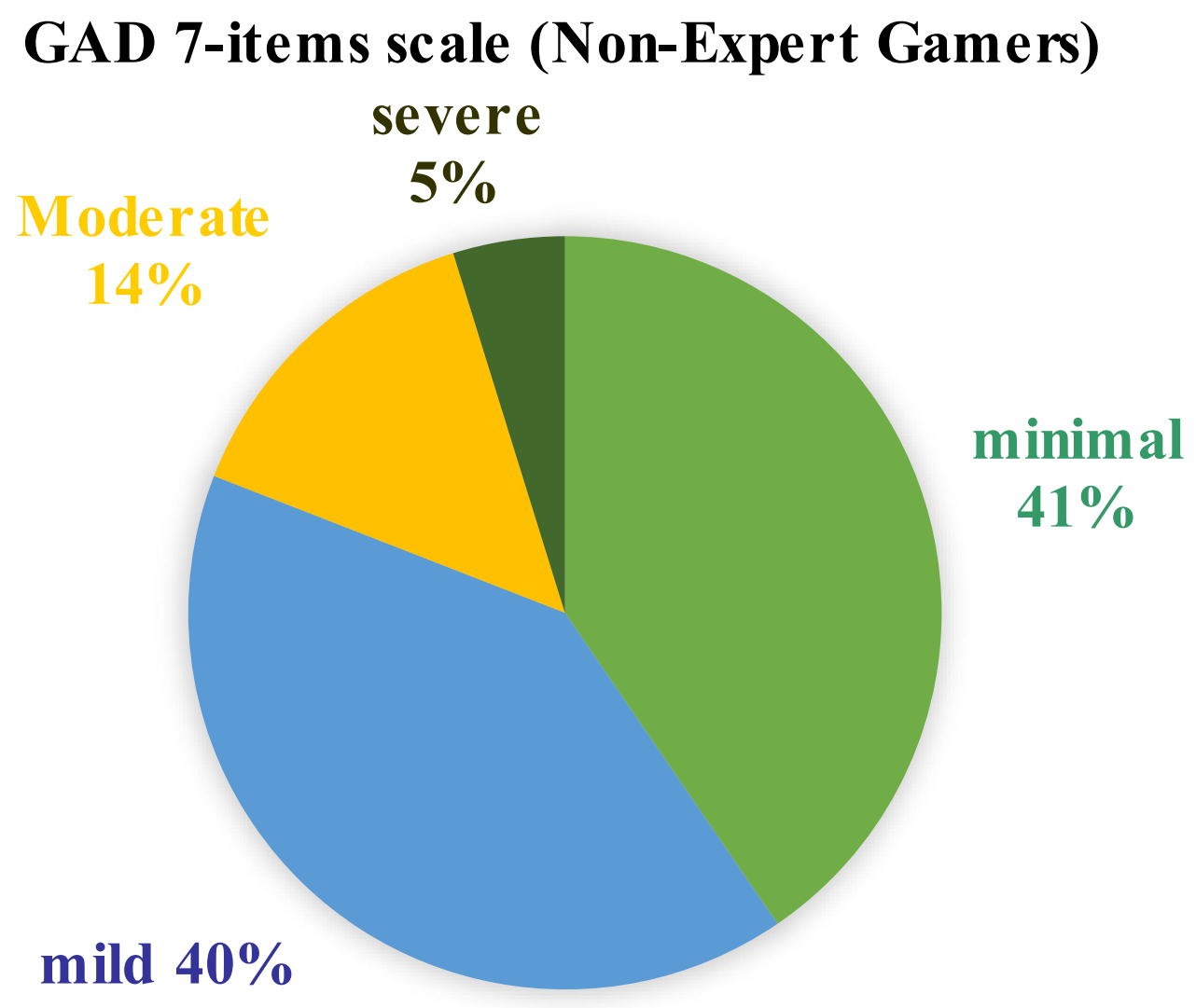

Figure $4 \mathrm{GAD}$ score for non-expert gamers.

explained by the nature of monotony and lack of exciting stimuli during the test. This makes both groups equal regarding attention span, with no superiority of gaming expertise in the improvements of this domain.

The second purpose of our contemporary study was to determine whether action video games were associated with elevated anxiety levels among healthy adolescents. We found no significant association between playing action video games and developing anxiety in expert or non-expert gamers $(\mathrm{p}=0.39)$. Overall, $87.3 \%$ of expert and $81 \%$ of non-expert gamers reported having minimal to mild anxiety symptoms, reflecting no direct relationship between anxiety and gaming regimens. These results are consistent with those of a previous review that observed the relationship between addictive use of social media and video games and psychiatric disorders; the results suggested that playing video games had no impact on anxiety (even for addicted video gamers) in terms of improvement or worsening, and more gamers were found to be depressed than anxious. ${ }^{25}$ A separate randomized controlled trial examined the effect of video games on adolescent anxiety symptoms; it showed a steeper decrease in personalized anxiety scores than in total anxiety scores. Two plausible explanations for the absence of impact were the duration of the intervention and the type of video game used. ${ }^{26}$ The first conflicting results were from a randomized controlled trial created by a multidisciplinary team of psychologists, clinicians, game designers, and children themselves; participants showed improvement in anxiety levels after maintaining the evidence-based therapeutic video games for 6 months. ${ }^{27}$ Another study indicated that female gamers experienced

Table 4 Sleepiness Level Comparison Between Expert and Non-Expert Gamers

\begin{tabular}{|l|l|l|l|l|l|}
\hline \multirow{2}{*}{ Gamers } & \multicolumn{2}{|l|}{ Score (\%) } & \multirow{2}{*}{ Total (\%) } & \multirow{2}{*}{ Fisher's Exact Test } & \multirow{2}{*}{ p-value } \\
\cline { 2 - 5 } & Normal Daytime Sleepiness & Excessive Daytime Sleepiness & & & \\
\hline Expert gamers & $47(85.5 \%)$ & $8(14.5 \%)$ & $55(56.7 \%)$ & 0.426 & \\
Non-expert gamers & $33(78.5 \%)$ & $9(21.5 \%)$ & $42(43.3 \%)$ & 0.268 & 0.377 \\
\hline
\end{tabular}


higher anxiety in comparison to males, but the reason behind that was unclear. ${ }^{28}$ Additional studies reported variability based on the type of video games, anxiety scales used, age, and gender. One 2019 study revealed that anxiety was a risk factor for gaming addiction symptoms, and players who had higher anxiety levels tended to have higher addiction scores. ${ }^{29}$ Finally, one study found a direct relationship between video game addiction and psychological factors such as depression, stress, and anxiety, ${ }^{30}$ and one additional study supported this finding. ${ }^{31}$

The effect of technology usage on human sleep is an ever-growing area of study. The third objective of the present study was to examine the effect of video games on sleep. Results showed that $85.5 \%$ of expert and $78.5 \%$ of non-expert video gamers reported normal daytime sleepiness, while the rest reported excessive daytime sleepiness. These results contradict the established results from existing literature stating that video games have a negative impact on sleep. ${ }^{32}$ The discrepancy between our results and those in the literature may be explained by several limitations and methodological means observed in literature. Although the effects of video games on sleep appears to be extensively studied, previous studies investigating this relationship are actually very limited. This may be the result of confusing the term "video gaming" with media use (television, personal computer, or Internet) rather than regarding these things as separate entities with different effects. ${ }^{3-35}$ Another limitation was the wide variety of methodological instruments and protocols used in each study, making it difficult to generalize and harmonize conclusions between them. ${ }^{32}$ Furthermore, many factors are linked to disturbances in sleeping patterns - specifically, the previous day's activities and increased arousal states. ${ }^{36}$ This highlights the importance of separating the effect of video gaming on sleep from those of concurrent factors in daily life.

There are some limitations within this study that should be considered. The study was conducted among a small group of participants with some similar characteristics, such as age. This fact may limit the generalizability of the results. We suggest applying the study to a larger population with comparable demographics.

\section{Conclusion}

This study showed that expert video gamers were significantly more attentive compared to non-expert video gamers. Additionally, attention span decreased gradually toward the end of the trials in both groups of gamers, indicating that attention is not sustained with time. Although most of the participants showed mild levels of anxiety, there was no association between the severity of gaming and either anxiety level or daytime sleepiness. This study assessed the effects of gaming on attention and concluded that attention can be altered by certain types of games. That is, certain types of games can be used as tools to alter higher cognitive brain functions, such as attention, especially in those members of society who suffer from attention disorders that affect their day-to-day performances. This study also calls for the development of targeted video games that benefit members of society with disabilities in cognitive function. Further studies examining different types of video games and their effects on higher cognitive functions are recommended. Regarding the effects of gaming on cognitive functions, the test we used to assess attention focused on reaction time and did not take more than 10 minutes for 1 participant to finish. In further research, more complex tests that measure other parameters of cognition might be used to give more detailed information about different cognitive domains affected by gaming behavior, rather than just attention. Although online questionnaires are commonly used in other studies in this field and are reported to be an effective method, the nature of online surveys has some limitations regarding the selfreporting of symptoms; mainly, this method not as accurate as an interview with a clinician.

\section{Data Sharing Statement}

All datasets used and/or analyzed during the current study are available from the corresponding author on reasonable request.

\section{Ethics Approval}

The protocol for this research was approved by the Ethics and Research Committee of the Imam Abdulrahman Bin Faisal University (IRB number: IRB-UGS-2020-01-358). 


\section{Informed Consent}

Written informed consent was obtained from the participants for obtaining their participation results to be published in this article.

\section{Acknowledgments}

We thank the students, Rajaei Ali and Saad Almutairi, from Imam Abdulrahman Bin Faisal University for helping to connect us to the participant students in the study.

\section{Author Contributions}

All listed authors: LB, NA, MA, FA, AA, KA, TA, and MS made a significant contribution to the work reported, whether that is in the conception, study design, execution, acquisition of data, analysis and interpretation, or in all these areas; took part in drafting, revising or critically reviewing the article; gave final approval of the version to be published; have agreed on the journal to which the article has been submitted; and agree to be accountable for all aspects of the work.

\section{Funding}

The authors declare that no outside funding was used for this research.

\section{Disclosure}

The authors declare that they have no conflicts of interest for this work.

\section{References}

1. Feng W, Ramo D, Chan S, Bourgeois J. Internet gaming disorder: trends in prevalence 1998-2016. Addict Behav. 2017;75:17-24. doi:10.1016/j. addbeh.2017.06.010

2. Farchakh Y, Haddad C, Sacre H, Obeid S, Salameh P, Hallit S. Video gaming addiction and its association with memory, attention and learning skills in Lebanese children. Child Adolesc Psychiatry Ment Health. 2020;14(1):46. doi:10.1186/s13034-020-00353-3

3. Qiu N, Ma W, Fan X, et al. Rapid improvement in visual selective attention related to action video gaming experience. Front Hum Neurosci. 2018;12:47. doi:10.3389/fnhum.2018.00047

4. Trisolini D, Petilli M, Daini R. Is action video gaming related to sustained attention of adolescents? Q J Exp Psychol. 2018;71(5):1033-1039. doi:10.1080/17470218.2017.1310912

5. Yen J-Y, Yeh Y-C, Wang P-W, Liu T-L, Chen -Y-Y, Ko C-H. Emotional regulation in young adults with internet gaming disorder. Int J Environ Res Public Health. 2017;15(1):30. doi:10.3390/ijerph15010030

6. Marino C, Canale N, Vieno A, Caselli G, Scacchi L, Spada M. Social anxiety and internet gaming disorder: the role of motives and metacognitions. J Behav Addict. 2020;9(3):617-628. doi:10.1556/2006.2020.00044

7. Wang C-Y, Wu Y-C, Su C-H, Lin P-C, Ko C-H, Yen J-Y. Association between internet gaming disorder and generalized anxiety disorder. $J$ Behav Addict. 2017;6(4):564-571. doi:10.1556/2006.6.2017.088

8. Fazeli S, Zeidi I, Lin C-Y, et al. Corrigendum to "depression, anxiety, and stress mediate the associations between internet gaming disorder, insomnia, and quality of life during the COVID-19 outbreak" [Addict. Behav. Rep. 12 (2020) 100307]. Addict Behav Rep. 2020;12:100322. doi:10.1016/j.abrep.2020.100322

9. Lam LT. Internet gaming addiction, problematic use of the internet, and sleep problems: a systematic review. Curr Psychiatry Rep. 2014;16(4):444. doi:10.1007/s11920-014-0444-1

10. An J, Sun Y, Wan Y, Chen J, Wang X, Tao F. Associations between problematic internet use and adolescents' physical and psychological symptoms. $J$ Addict Med. 2014;8(4):282-287. doi:10.1097/ADM.0000000000000026

11. Wong HY, Mo HY, Potenza MN, et al. Relationships between severity of internet gaming disorder, severity of problematic social media use, sleep quality and psychological distress. Int J Environ Res Public Health. 2020;17(6):1879. doi:10.3390/ijerph17061879

12. Chisholm JD, Hickey C, Theeuwes J, Kingstone A. Reduced attentional capture in action video game players. Atten Percept Psychophys. 2010;72 (3):667-671. doi:10.3758/app.72.3.667

13. Mueller ST, Piper BJ. The Psychology Experiment Building Language (PEBL) and PEBL test battery. J Neurosci Methods. 2014;222:250-259. doi:10.1016/j.jneumeth.2013.10.024

14. Spitzer RL, Kroenke K, Williams JBW, Löwe B. A brief measure for assessing generalized anxiety disorder: the GAD-7. Arch Intern Med. 2006;166(10):1092-1097. doi:10.1001/archinte.166.10.1092

15. Terrill AL, Hartoonian N, Beier M, Salem R, Alschuler K. The 7-item generalized anxiety disorder scale as a tool for measuring generalized anxiety in multiple sclerosis. Int J MS Care. 2015;17(2):49-56. doi:10.7224/1537-2073.2014-008

16. Johns MW. A new method for measuring daytime sleepiness: the Epworth Sleepiness Scale. Sleep. 1991;14(6):540-545. doi:10.1093/sleep/ 14.6.540

17. Packard A, Bautista R, Smotherman C, Gautham S. Gender differences in Epworth Sleepiness Scale revealed by paired patient-spouse scoring. Epilepsy Behav. 2021;114:(Pt A):107272. doi:10.1016/j.yebeh.2020.207272

18. Mishra J, Bavelier D, Gazzaley A. How to assess gaming-induced benefits on attention and working memory. Games Health J. 2012;1(3):192-198. doi: $10.1089 / \mathrm{g} 4 \mathrm{~h} .2011 .0033$ 
19. Dye MWG, Green CS, Bavelier D. Increasing speed of processing with action video games. Curr Dir Psychol Science. 2009;18(6):321-326. doi:10.1111/j.1467-8721.2009.01660.x

20. Orosy-Fildes C, Allan RW. Psychology of computer use: XII. videogame play: human reaction time to visual stimuli. Percept Mot Skills. 1989;69 (1):243-247. doi:10.2466/pms.1989.69.1.243

21. Pardina Torner H, Carbonell X, Castejón M. A comparative analysis of the processing speed between video game players and non-players. Aloma. 2019;37(1):13-20. doi:10.51698/aloma.2019.37.1.13-20

22. Bavelier D, Green C. Enhancing attentional control: lessons from action video games. Neuron. 2019;104(1):147-163. doi:10.1016/j. neuron.2019.09.031

23. Franceschini S, Gori S, Ruffino M, Viola S, Molteni M, Facoetti A. Action video games make dyslexic children read better. Curr Biol. 2013;23 (6):462 466. doi:10.1016/j.cub.2013.01.044

24. Rosser J. The impact of video games on training surgeons in the 21st century. Arch Surg. 2007;142(2):181-186. doi:10.1001/archsurg.142.2.181

25. Andreassen CS, Billieux J, Griffiths MD, et al. The relationship between addictive use of social media and video games and symptoms of psychiatric disorders: a large-scale cross-sectional study. Psychol Addict Behav. 2016;30(2):252-262. doi:10.1037/adb0000160

26. Scholten H, Malmberg M, Lobel A, Engels RCME, Granic I. A randomized controlled trial to test the effectiveness of an immersive 3D video game for anxiety prevention among adolescents. PLoS One. 2016;11(1):e0147763. doi:10.1371/journal.pone.0147763

27. Wols A, Lichtwarck-Aschoff A, Schoneveld EA, Granic I. In-game play behaviours during an applied video game for anxiety prevention predict successful intervention outcomes. J Psychopathol Behav Assess. 2018;40(4):655-668. doi:10.1007/s10862-018-9684-4

28. Ohannessian CM. Video game play and anxiety during late adolescence: the moderating effects of gender and social context. $J$ Affect Disord. 2018;226:216-219. doi:10.1016/j.jad.2017.10.009

29. Adams BLM, Stavropoulos V, Burleigh TL, Liew LWL, Beard CL, Griffiths MD. Internet gaming disorder behaviors in emergent adulthood: a pilot study examining the interplay between anxiety and family cohesion. Int J Ment Health Addict. 2018;17(4):828-844. doi:10.1007/s11469-018-9873-0

30. Loton D, Borkoles E, Lubman D, Polman R. Video game addiction, engagement and symptoms of stress, depression and anxiety: the mediating role of coping. Int J Ment Health Addict. 2015;14(4):565-578. doi:10.1007/s11469-015-9578-6

31. González-Bueso V, Santamaría JJ, Fernández D, Merino L, Montero E, Ribas J. Association between internet gaming disorder or pathological video-game use and comorbid psychopathology: a comprehensive review. Int J Environ Res Public Health. 2018;15(4):668. doi:10.3390/ ijerph15040668

32. Peracchia S, Curcio G. Exposure to video games: effects on sleep and on post-sleep cognitive abilities. A sistematic review of experimental evidences. Sleep Sci. 2018;11(4):302-314. doi:10.5935/1984-0063.20180046

33. Brunetti VC, O’Loughlin EK, O’Loughlin J, Constantin E, Pigeon É. Screen and nonscreen sedentary behavior and sleep in adolescents. Sleep Health. 2016;2(4):335-340. doi:10.1016/j.sleh.2016.09.004

34. Arrona-Palacios A. High and low use of electronic media during nighttime before going to sleep: a comparative study between adolescents attending a morning or afternoon school shift. J Adolesc. 2017;61:152-163. doi:10.1016/j.adolescence.2017.10.009

35. Higuchi S, Motohashi Y, Liu Y, Maeda A. Effects of playing a computer game using a bright display on presleep physiological variables, sleep latency, slow wave sleep and REM sleep. J Sleep Res. 2005;14(3):267-273. doi:10.1111/j.1365-2869.2005.00463.x

36. Chang A-M, Aeschbach D, Duffy JF, Czeisler CA. Evening use of light-emitting eReaders negatively affects sleep, circadian timing, and nextmorning alertness. Proc Natl Acad Sci U S A. 2015;112(4):1232-1237. doi:10.1073/pnas.1418490112

\section{Publish your work in this journal}

Psychology Research and Behavior Management is an international, peer-reviewed, open access journal focusing on the science of psychology and its application in behavior management to develop improved outcomes in the clinical, educational, sports and business arenas. Specific topics covered in the journal include: Neuroscience, memory and decision making; Behavior modification and management; Clinical applications; Business and sports performance management; Social and developmental studies; Animal studies. The manuscript management system is completely online and includes a very quick and fair peer-review system, which is all easy to use. Visit http://www.dovepress.com/testimonials.php to read real quotes from published authors.

Submit your manuscript here: https://www.dovepress.com/psychology-research-and-behavior-management-journal 